\title{
Absence of Spontaneous Spin Symmetry Breaking in 1D and 2D Quantum Ferromagnetic Systems with Bilinear and Biquadratic Exchange Interactions
}

\author{
Roberto Zivieri ${ }^{1,2}$ \\ 1 Department of Mathematical and Computer Sciences, Physical Sciences and Earth Sciences, \\ University of Messina, 98166 Messina, Italy; roberto.zivieri@unife.it \\ 2 Istituto Nazionale di Alta Matematica (INdAM), Piazzale Aldo Moro 5, 00185 Rome, Italy
}

Received: 20 November 2020; Accepted: 9 December 2020; Published: 11 December 2020

\begin{abstract}
Some measurements have shown that the second-order exchange interaction is non-negligible in ferromagnetic compounds whose microscopic interactions are described by means of half-odd integer quantum spins. In these spin systems the ground state is either ferromagnetic or antiferromagnetic when the bilinear exchange interaction is dominant. Instead, in ferromagnetic systems characterized by bilinear and biquadratic exchange interactions of comparable magnitude, the energy minimum occurs when spins are in a canting ground-state. To this aim, a one-dimensional (1D) quantum spin chain and a two-dimensional (2D) lattice of quantum spins subjected to periodic boundary conditions are modeled via the generalized quantum Heisenberg Hamiltonian containing, in addition to the isotropic and short-range bilinear exchange interaction of the Heisenberg type, a second-order interaction, the isotropic and short-range biquadratic exchange interaction between nearest-neighbors quantum spins. For these 1D and 2D quantum systems a generalization of the Mermin-Wagner-Hohenberg theorem (also known as Mermin-Wagner-Berezinksii or Coleman theorem) is given. It is demonstrated, by means of quantum statistical arguments, based on Bogoliubov's inequality, that, at any finite temperature, (1) there is absence of long-range order and that (2) the law governing the vanishing of the order parameter is the same as in the bilinear case for both 1D and 2D quantum ferromagnetic systems. The physical implications of the absence of a spontaneous spin symmetry breaking in 1D spin chains and 2D spin lattices modeled via a generalized quantum Heisenberg Hamiltonian are discussed.
\end{abstract}

Keywords: ferromagnetic chain; 2D ferromagnetic lattice; spin Hamiltonian; double commutator; Bogoliubov inequality; Hohenberg-Mermin-Wagner theorem; biquadratic exchange interaction; continuous spin symmetry; canting ground-state

\section{Introduction}

The concept of long-range order is crucial to characterize phase transitions taking place in physical systems as a function of temperature and described by means of an order parameter [1]. This aspect acquires great importance for phase transitions undergoing in magnetic systems that represent the majority of phase transitions in condensed matter physics. Importantly, the appearance of long-range order is the manifestation of the occurrence of a phase transition and is related to a continuous or a discrete broken symmetry in magnetic systems depending on the model (either quantum or classical) used to describe the underlying physics of the phase transition (e.g., Ising model, Potts model, XY model, Heisenberg model, etc.) [1]. Undoubtedly, the isotropic Heisenberg model, both in its original quantum version and in its classical counterpart, represents one of the most powerful physical models applied to magnetic systems undergoing phase transitions where short-range interactions prevail. Its isotropic 
Hamiltonian allows to easily catching the physics of the magnetic system under study. In this respect, the Hohenberg-Mermin-Wagner theorem [2-4] (also known as Mermin-Wagner-Berezinskii or Coleman theorem [5]) represents a hallmark in the field of condensed matter physics. Hohenberg [2] used the Bogoliubov inequality [6] to show that, at finite temperature, there is no long-range order in 1D and 2D Bose and Fermi systems. At the same time, Mermin and Wagner [3] considered the case of spontaneous magnetization in the isotropic exchange Heisenberg model showing that there cannot be a spontaneous symmetry breaking at finite temperature $T$ for 1D and 2D ferromagnets with either a ferromagnetic (F) or an antiferromagnetic (AF) ground-state. Mermin extended the results to ferromagnets modeled via the classical Heisenberg model showing that a classical Bogoliubov-type inequality holds using the Poisson brackets ruling out phase transitions for the 'classical' limit of the Heisenberg model, i.e., in the infinite-spin limit [7]. A proof of the Hohenberg-Mermin-Wagner theorem for systems with film geometries has been given two decades ago for the Heisenberg, Hubbard, s-f and Kondo-lattice models by Gelfert and Nolting [8]. In particular, in this work Gelfert and Nolting have proposed an extension of the validity of the Mermin-Wagner system to the periodic Anderson model with film geometry and to a superconducting pairing mechanism in Hubbard films [8] and later have reviewed different cases of absence of finite-temperature phase transitions in low-dimensional many-body models [9]. The extension to an excitonic insulating state was made by Walker who used the Bogoliubov inequality to rule out the possibility of a phase transition in 1D and 2D systems [10]. More recently, a generalization of the Hohenberg-Mermin-Wagner theorem to $1 \mathrm{D}$ and 2D quantum ferromagnetic systems in the presence of long-range dipolar interactions was made [11], while Loss et al. [12] proved the absence of spontaneous magnetic order in a system of lattice spins coupled to itinerant and interacting charge carriers.

On the other hand, in the 1960s, Harris and Owen showed that it was necessary to add a biquadratic exchange interaction to explain the energy level spectrum of Mn pairs in MgO compounds [13,14]. On this basis, Huang and Orbach investigated the biquadratic superexchange arising from a balancing mechanism between elastic and exchange forces in Mn pairs [15]. In the 1970s, high temperature series expansions for dipole and quadrupole susceptibilities for arbitrary lattices modeled by means of isotropic bilinear and biquadratic pair interaction for spin-1 ferromagnetic systems were derived [16]. A theoretical model dealing with a unified exchange picture was developed for classical ferromagnets in [17] showing that it is natural, for a complete phenomenological description of exchange interactions between ferromagnetic films separated by a paramagnetic spacer layer, to include higher-order contributions in addition to the usual first-order Heisenberg term. More recently, it has been shown that the biquadratic interaction must be included phenomenologically to explain the phase diagram of Fe ferromagnetic bilayers with easy axis anisotropy antiferromagnetically coupled via different spacers (e.g., $\mathrm{Cr}, \mathrm{Al}, \ldots$ ) [18]. It has been proved, both experimentally and theoretically, that magnetizations of the two Fe ferromagnets can be either in a canted ground-state due to the balancing between the bilinear and the biquadratic exchange coupling or in a $\pi / 2$ ground-state when the biquadratic exchange interaction between ferromagnetic layers prevails over the bilinear one in systems with four-fold anisotropy [18]. Later, the study of the excitation spectra of $\mathrm{Mn}_{2}$ dimers in $\mathrm{CsMn}_{0.28} \mathrm{Mg}_{0.72} \mathrm{Br}_{3}$ by using the inelastic neutron scattering technique has confirmed that the biquadratic exchange stems from a local exchange striction mechanism observed taking into account a magnetoelastic effect [19]. These recent findings and observations have stimulated several theoretical studies about the role played by the biquadratic contribution on the dynamical properties of ferromagnetic films coupled via antiferromagnetic Heisenberg exchange coupling.

On this basis, it is interesting to extend the Hohenberg-Mermin-Wagner theorem to quantum systems modeled via a generalized quantum Heisenberg Hamiltonian including both isotropic bilinear and biquadratic exchange short-range interactions. This is done for the case of a 1D chain of half-odd integer spins and for a 2D square lattice system of half-odd integer spins with periodic boundary conditions. First, it is rigorously proved that the generalized Hamiltonian modeling these systems is invariant under the spin rotation generator showing that it commutes with the spin rotation generator. 
Then, it is rigorously shown, according to thermodynamics arguments, that there is absence of long-range order at finite $T$ (spontaneous magnetization in ferromagnets and sublattice magnetization in antiferromagnets) in 1D and 2D magnetic systems with both bilinear and biquadratic exchange terms. For 1D quantum spin chains and 2D quantum spin lattices it is proved that the order parameter vanishes as the external magnetic field goes to zero following the same law of the Hohenberg-Mermin-Wagner theorem where only the bilinear exchange term was included in the quantum Heisenberg Hamiltonian. Hence, there is no spontaneous spin symmetry breaking at any $T$ in quantum spin systems modeled by means of a generalized quantum Heisenberg Hamiltonian including the biquadratic exchange interaction. This result is not in conflict with the occurrence of phase transitions at finite $T$ proposed by Stanley and Kaplan for 2D lattices with nearest-neighbor ferromagnetic Heisenberg interactions [20] and marked by a divergence of the susceptibility [21]. The proof of the existence of such a phase transition was given by Frölich and Spencer [22] who discussed the Berezinski-Kosterlitz-Thouless transition in a class of 2D models including the plane rotator and the Coulomb gas. This proof was based on the general method for demonstrating the existence of phase transitions in anisotropic lattice spin systems developed by Frölich and Lieb [23]. It is shown that the vanishing of the order parameter at any $T$ not only rules out either the $\mathrm{F}$ or the AF ground-state but also the canting $(\mathrm{C})$ ground-state occurring when the biquadratic exchange interaction is comparable to the bilinear one. The canting angle is the angle forming between adjacent spins that reduces its amplitude with increasing the amplitude of the external magnetic field. This is an important result because a novel ground-state giving no long-range order is added to the phase diagram of a quantum ferromagnet.

Note that, in this respect, there is a proof given by Thorpe in the 70s who demonstrated the absence of long-range order at finite $T$ in $1 \mathrm{D}$ and $2 \mathrm{D}$ ferromagnetic lattices modeled by a generalized quantum Heisenberg Hamiltonian containing isotropic spin-spin exchange interactions to all orders [24]. This proof was obtained by using the Bogoliubov's inequality and developing a mathematical approach based on writing terms of the spin equivalent of the spherical harmonics. A few years later, Krzemiński used the same mathematical method and came to similar conclusions demonstrating the absence of long-range order also in multi-sublattice systems [25]. The absence of off-diagonal long-range order and of long-range order was demonstrated in 2D ferromagnetic lattices described via the Heisenberg model by Suzuki [26] who also showed that, in systems with higher spins leading to a different definition of order parameter, off-diagonal long-range order can exist and the order parameter can remain finite. Finally, the absence of spontaneous breaking of continuous and internal symmetries, of crystalline ordering in 2D systems and uniqueness of equilibrium states was proved by Frölich and Pfister [27] according to a unified approach based on Araki's relative entropy concept. However, for the systems studied in [24-27], the analysis was mainly mathematical and the physical effects of the biquadratic exchange interaction were not investigated.

\section{Generalized Quantum Heisenberg Hamiltonian}

In this Section, the absence of long-range order in a 1D quantum chain of integer spins and in a 2D lattice of $N$ quantum spins modeled via the generalized Heisenberg model that includes both the isotropic bilinear and biquadratic short-range exchange interactions is rigorously proved. The proof of the generalization of the Hohenberg-Mermin-Wagner theorem, valid for quantum ferromagnetic systems with either ferro- or antiferromagnetic coupling in $1 \mathrm{D}$ and 2D [2,3], to the case of a generalized quantum Heisenberg Hamiltonian is given by using the Bogoliubov's inequality. The generalization of the Hohenberg-Mermin-Wagner theorem is preceded by the proof of the rotational invariance of the generalized quantum exchange Hamiltonian under the spin rotation operator.

\subsection{Generalized Quantum Heisenberg Hamiltonian: Bilinear and Biquadratic Exchange Interactions}

In this subsection, the key physical quantities that can be applied to both $1 \mathrm{D}$ and 2D quantum ferromagnetic systems of spins modeled via a generalized quantum Heisenberg Hamiltonian are 
defined. First, let us define a spin-density wave, a complex quantity characterized by a wave vector $q$ propagating along the $z$-direction by means of the operator [28]:

$$
S_{\boldsymbol{q}}^{z}=\sum_{i=0}^{N-1} e^{i \boldsymbol{q} \cdot \boldsymbol{R}_{i}} S_{i}^{z}
$$

with $\boldsymbol{R}_{i}$ the $i$ th position vector with the index $i=0,1, \ldots N-1$ ranging over the $N$ spins and $S_{i}^{z}$ the $z$-component of the spin quantum operator $\mathbf{S}_{i}$. The generalized quantum Heisenberg Hamiltonian for half-odd integer spin chains takes the form:

$$
\mathcal{H}=\frac{1}{2} \sum_{<i, j>} J_{i j}^{(1)} \mathbf{S}_{i} \cdot \mathbf{S}_{j}+\frac{1}{2} \sum_{<i, j>} J_{i j}^{(2)}\left(\mathbf{s}_{i} \cdot \mathbf{S}_{j}\right)^{2}-B_{\text {ext }} S_{q}^{z}
$$

where, on the second member, the first term is the isotropic bilinear exchange term, the second term is the isotropic biquadratic exchange term and the last term is the contribution proportional to the external magnetic field. Specifically, $\forall$ ij pair of nearest-neighbors $J_{i j}{ }^{(1)}$ is the bilinear exchange constant (in energy units) with $J_{i j}{ }^{(1)}=J_{j i}{ }^{(1)}\left(J_{i j}{ }^{(1)}>0(<0)\right.$ corresponds to the $\mathrm{AF}(\mathrm{F})$ ground-state for the quantum Heisenberg Hamiltonian) and $J_{i j}{ }^{(2)}$, either $>0$ or $<0$, is the biquadratic exchange constant (in energy units) with $J_{i j}{ }^{(2)}=J_{j i}{ }^{(2)}$. Both $J_{i j}{ }^{(1)}$ and $J_{i j}{ }^{(2)}$ have the lattice translational symmetry, $\sum_{\langle i, j\rangle}$ is the sum over the nearest-neighbours $j \forall i$ with the factor $\frac{1}{2}$ to not count twice the interaction, $\mathbf{S}_{i}=\left(S_{i}{ }^{x}, S_{i}{ }^{y}\right.$, $\left.S_{i}^{z}\right)$ and $\mathbf{S}_{j}=\left(S_{j}^{x}, S_{j}^{y}, S_{j}^{z}\right)$ are the spin operators and $B_{\text {ext }}>0$ the amplitude of the external magnetic field (in energy units). Note that, unlike the usual convention, in the Hamiltonian of Equation (2) the minus sign in front of both the bilinear and biquadratic exchange terms has been incorporated in $J_{i j}{ }^{(1)}$ and $J_{i j}{ }^{(2)}$, respectively so that the signs of $J_{i j}{ }^{(1)}$ and $J_{i j}{ }^{(2)}$ must be taken as opposite to those of the usual convention.

The condition for short-range interactions on the bilinear and biquadratic terms reads:

$$
\bar{J}^{(1)}=\frac{1}{2 N} \sum_{<i, j>}\left|J_{i j}^{(1)}\right|\left|\boldsymbol{R}_{i}-\boldsymbol{R}_{j}\right|^{2}<\infty \quad \bar{J}^{(2)}=\frac{1}{2 N} \sum_{<i, j>}\left|J_{i j}^{(2)}\right|\left|\boldsymbol{R}_{i}-\boldsymbol{R}_{j}\right|^{2}<\infty
$$

must be fulfilled. The corresponding spin-density wave along the $x$-direction characterized by a wave vector $k$ is:

$$
S_{k}^{x}=\sum_{i=0}^{N-1} e^{i k \cdot R_{i}} S_{i}^{x}
$$

and the spin-density wave along the $y$-direction characterized by a wave vector $k+q$ takes the form:

$$
S_{\boldsymbol{k}+\boldsymbol{q}}^{y}=\sum_{i=0}^{N-1} e^{i(\boldsymbol{k}+\boldsymbol{q}) \cdot \boldsymbol{R}_{i}} S_{i}^{y}
$$

From the Schwartz inequality, it is possible to derive the Bogoliubov inequality. This inequality represents the starting point to show the absence of long-range order at any finite $T$ for any $1 \mathrm{D}$ and 2D quantum spins magnetic system modeled by a generalized quantum Heisenberg Hamiltonian. The Bogoliubov inequality reads [28]:

$$
\left|\left\langle\left[S_{-\boldsymbol{k}^{\prime}}^{x} S_{\boldsymbol{k}+\boldsymbol{q}}^{y}\right]\right\rangle\right|^{2} \leq \frac{N}{k_{\mathrm{B}} T}\left\langle S_{\boldsymbol{k}+\boldsymbol{q}}^{y} S_{-\boldsymbol{k}-\boldsymbol{q}}^{y}\right\rangle D(\boldsymbol{k})
$$

where $k_{\mathrm{B}}$ is the Boltzmann constant, $T$ is the temperature, $\left[S_{-k^{\prime}}^{x}, S_{k+q}^{y}\right]$ is the commutator between $x$-and $y$-spin density waves of wave vectors $-k$ and $k+\boldsymbol{q}$, respectively, $\left\langle S_{\boldsymbol{k}+\boldsymbol{q}}^{y} S_{-\boldsymbol{k}-\boldsymbol{q}}^{y}\right\rangle$ is the equal-time 
correlation function of the $y$-component of the spin density wave operator with $<\ldots>$ denoting the statistical (thermal) average, $D(k)=\frac{1}{N}<\left[S_{-k^{\prime}}^{x}\left[\mathcal{H}, S_{k}^{x}\right]\right]>$ is the double commutator defined as a statistical average. The double commutator (in energy units) includes the Hamiltonian of the generalized Heisenberg model and the spin-density wave operator giving thus information about the dynamical properties of the quantum ferromagnetic system under study.

By means of Equation (1) the magnetization per site that plays the role of a complex order parameter for the generalized 1D and 2D quantum Heisenberg Hamiltonian $\mathcal{H}=\mathcal{H}\left(B_{\text {ext }}\right)$ can be defined as a statistical (thermal) average of the spin-density wave $S_{q}^{z}$ along the $z$-direction divided by the number $N$ of quantum spins [28]:

$$
m_{\boldsymbol{q}}=\frac{1}{N Z} \operatorname{Tr}\left[e^{-\mathcal{H}\left(B_{\mathrm{ext}}\right) /\left(k_{B} T\right)} S_{\boldsymbol{q}}^{z}\right]
$$

where $\operatorname{Tr}$ is the trace and $Z=\operatorname{Tr}\left[e^{-\mathcal{H}\left(B_{\mathrm{ext}}\right) / k_{B} T}\right]$ is the partition function. The order parameter defined in Equation (7) is a key quantity to study phase transitions. It measures the degree of order of a physical system undergoing a phase transition. It can be defined for both a classical and a quantum system as in the present case. It is thus crucial to start from this fundamental physical quantity to prove the absence of long-range order. Equation (7) expresses a statistical (thermal) average of the spin density wave propagating along the $z$-direction that represents a low-energy ordered state of the quantum spin system. It can be regarded as the average $z$-component of the spin operator expressing in this case the net "magnetization" per site in a F state, AF state or C ground-state. For a ferromagnet the net "magnetization" refers to the whole lattice, while for an antiferromagnet it refers to each sublattice. The order parameter is in general associated with the ordered phase resulting from a symmetry breaking phase transition but it can be defined also in phase transitions with no symmetry breaking.

The order parameter $m_{q}$ becomes a real quantity in quantum lattices that are symmetric under reflection about the origin so that the operator $S_{q}^{z}$ is Hermitian. Owing to the definition of Equation (7), the commutator between the $x$ and the $y$ spin-density wave components defined by Equations (4) and (5), respectively reads:

$$
\left\langle\left[S_{-\boldsymbol{k}^{\prime}}^{x} S_{\boldsymbol{k}+\boldsymbol{q}}^{y}\right]\right\rangle=i\left\langle S_{\boldsymbol{q}}^{z}\right\rangle=i N m_{\boldsymbol{q}}
$$

Explicitly, the equal-time correlation function of the $y$-components of the spin density wave operators reads:

$$
\left\langle S_{\boldsymbol{k}+\boldsymbol{q}}^{y} S_{-\boldsymbol{k}-\boldsymbol{q}}^{y}\right\rangle=N S^{y y}(\boldsymbol{k}+\boldsymbol{q})
$$

with:

$$
S^{y y}(\boldsymbol{k}+\boldsymbol{q})=\lim _{B_{\mathrm{ext}} \rightarrow 0^{+}}\left[\frac{1}{N Z} \operatorname{Tr}\left[e^{-\mathcal{H}\left(B_{\mathrm{ext}}\right) /\left(k_{B} T\right)} S_{\boldsymbol{k}+\boldsymbol{q}^{y}}^{y} S_{-\boldsymbol{k}-\boldsymbol{q}}^{y}\right]\right]
$$

In the following, basing on Equations (1)-(6), it is first proved the absence of long-range order for a 1D quantum Heisenberg chain of spins and then for a 2D quantum Heisenberg lattice of spins (with either ferro-or antiferromagnetic bilinear exchange interaction) in the presence of a biquadratic interaction (generalized quantum Heisenberg Hamiltonian).

\subsection{Rotational Symmetry and Invariance of the Generalized Quantum Exchange Hamiltonian}

In this subsection, the rotational symmetry and the invariance of the generalized quantum exchange Hamiltonian under a continuous spin rotation operation is proved. This Hamiltonian includes both the isotropic bilinear and biquadratic exchange interactions for vanishing external magnetic field. First, we consider the case of the 1D quantum chain of spins.

Let us consider a chain of $N$ integer spins (one-dimensional quantum system) on a 1D spin lattice with each site characterized by a spin operator $\mathbf{S}_{i}(i=0,1,2, \ldots, N-1)$ and characterized by nearest-neighbors interactions. The generalized quantum Heisenberg Hamiltonian for a $1 \mathrm{D}$ quantum 
chain of spins is written starting from the general Equation (2) by setting $j=i+1$ with $i+1$ the nearest-neighbor of the $i$ th spin and takes the form:

$$
\mathcal{H}^{1 \mathrm{D}}=\sum_{i=0}^{N-1} J_{i+1}^{(1)} \mathbf{S}_{i} \cdot \mathbf{S}_{i+1}+\sum_{i=0}^{N-1} J_{i+1}^{(2)}\left(\mathbf{S}_{i} \cdot \mathbf{S}_{i+1}\right)^{2}-B_{\mathrm{ext}} S_{\boldsymbol{q}}^{z}
$$

The proof consists of demonstrating that the spin rotation generator commutes with the generalized exchange Hamiltonian of the 1D quantum chain subjected to the boundary condition $\mathbf{S}_{N}=\mathbf{S}_{0}$ :

$$
\mathcal{H}_{\text {exch }}^{1 \mathrm{D}}=\mathcal{H}_{\text {exch }}^{(1) 1 \mathrm{D}}+\mathcal{H}_{\text {exch }}^{(2) 1 \mathrm{D}}
$$

with $\mathcal{H}_{\text {exch }}^{(1) 1 \mathrm{D}}=\sum_{i=0}^{N-1} J_{i+1}^{(1)} \mathbf{S}_{i} \cdot \mathbf{S}_{i+1}$ and $\mathcal{H}_{\mathrm{exch}}^{(2) 1 \mathrm{D}}=\sum_{i=0}^{N-1} J_{i i+1}^{(2)}\left(\mathbf{S}_{i} \cdot \mathbf{S}_{i+1}\right)^{2}$, namely

$$
\left[S^{\alpha}, \mathcal{H}_{\text {exch }}^{1 \mathrm{D}}\right]=0
$$

being the spin rotation generator defined, $\forall \alpha$ spin component, as:

$$
S^{\alpha}=\sum_{i=0}^{N-1} S_{i}^{\alpha}
$$

with $\alpha=x, y, z$. The rotation operator in spin space or spin rotation operator $U_{R}(\hat{n}, \theta)$ expanded to the first order for small angular rotations is defined via $\hat{n}$ denoting the rotation axis given by the direction of $\hat{n}$ and by means of a rotation angle $\theta$ as:

$$
U_{R}(\hat{\boldsymbol{n}}, \theta) \approx 1-i \hat{\boldsymbol{n}} \cdot \mathbf{S}, \hat{\boldsymbol{n}}=\left(n_{x}, n_{y}, n_{z}\right), \mathbf{S}=\left(S^{x}, S^{y}, S^{z}\right)
$$

The angle $\theta$ parametrizes the rotations in a unique way for the values $\theta<2 \pi$.

Let us prove that the generalized quantum Heisenberg Hamiltonian is invariant under a continuous $\mathrm{O}(3)$ rotational symmetry in spin space. As already anticipated in Equation (13), it is enough to prove the commutativity of the generalized exchange Hamiltonian with the spin rotation generator $S^{\alpha}(\forall \alpha$ spin component) appearing in the rotation operator $U_{R}(\hat{n}, \theta)$ of Equation (15) and the proof is general (for more details about this point, see Section 3).

The commutator of Equation (13) can be decomposed into two commutators $C_{1}$ and $C_{2}$, namely $\left[S^{\alpha}, \mathcal{H}_{\text {exch }}^{1 \mathrm{D}}\right]=C_{1}+C_{2}$. Here, $C_{1}=\left[S^{\alpha}, \mathcal{H}_{\text {exch }}^{(1)} 1 \mathrm{D}\right]$ and $C_{2}=\left[S^{\alpha}, \mathcal{H}_{\text {exch }}^{(2)} 1 \mathrm{D}\right]$. Substituting $S^{\alpha}$ expressed by Equation (14) in the commutator $C_{1}$ we get: $C_{1}=\sum_{i=0}^{N-1} \sum_{j=0}^{N-1}\left\{J_{j j+1}^{(1)}\left(\left[S_{i}^{\alpha} \delta_{i j}, \mathbf{S}_{j} \cdot \mathbf{S}_{j+1}\right]+\left[S_{i}^{\alpha} \delta_{i j+1}, \mathbf{S}_{j} \cdot \mathbf{S}_{j+1}\right]\right)\right\}$. From the property of the Kronecker delta, viz. $\delta_{i j}=1$ for $i=j$ and $\delta_{i j}=0$ for $i \neq j$ and $\delta_{i j+1}=1$ for $i=j+1$ and $\delta_{i j+1}=0$ for $i \neq j+1$ :

$$
C_{1}=\sum_{j=0}^{N-1}\left\{J_{j j+1}^{(1)}\left(\left[S_{j}^{\alpha}, \mathbf{S}_{j} \cdot \mathbf{S}_{j+1}\right]+\left[S_{j+1}^{\alpha}, \mathbf{S}_{j} \cdot \mathbf{S}_{j+1}\right]\right)\right\}
$$
$=0$.

Taking into account that $\left[S_{j+1}^{\alpha}, \mathbf{S}_{j} \cdot \mathbf{S}_{j+1}\right]=-\left[S_{j}^{\alpha}, \mathbf{S}_{j} \cdot \mathbf{S}_{j+1}\right]$ is fulfilled $\forall \alpha$, it is the commutator $C_{1}$ Analogously $C_{2}=\sum_{i=0}^{N-1} \sum_{j=0}^{N-1}\left\{J_{j j+1}^{(2)}\left(\left[S_{i}^{\alpha} \delta_{i j},\left(\mathbf{S}_{j} \cdot \mathbf{S}_{j+1}\right)^{2}\right]+\left[S_{i}^{\alpha} \delta_{i j+1},\left(\mathbf{S}_{j} \cdot \mathbf{S}_{j+1}\right)^{2}\right]\right)\right\}$ can be written as:

$$
C_{2}=\sum_{j=0}^{N-1}\left\{J_{j j+1}^{(2)}\left(\left[S_{j}^{\alpha},\left(\mathbf{S}_{j} \cdot \mathbf{S}_{j+1}\right)^{2}\right]+\left[S_{j+1}^{\alpha},\left(\mathbf{S}_{j} \cdot \mathbf{S}_{j+1}\right)^{2}\right]\right)\right\}
$$


The two commutators inside the round parentheses on the right-hand side can be rewritten, according to a commutator identity, as:

$$
\left[S_{t}^{\alpha},\left(\mathbf{S}_{j} \cdot \mathbf{S}_{j+1}\right)^{2}\right]=\left[S_{t}^{\alpha}, \mathbf{S}_{j} \cdot \mathbf{S}_{j+1}\right]\left(\mathbf{S}_{j} \cdot \mathbf{S}_{j+1}\right)+\left(\mathbf{S}_{j} \cdot \mathbf{S}_{j+1}\right)\left[S_{t}^{\alpha}, \mathbf{S}_{j} \cdot \mathbf{S}_{j+1}\right]
$$

with $t=j, j+1$.

Taking into account that, according to Equation (18), $\left[S_{j+1^{\prime}}^{\alpha}\left(\mathbf{S}_{j} \cdot \mathbf{S}_{j+1}\right)^{2}\right]=-\left[S_{j}^{\alpha},\left(\mathbf{S}_{j} \cdot \mathbf{S}_{j+1}\right)^{2}\right] \forall \alpha$, it is the commutator $C_{2}=0$. Hence, it has been proved that $\left[S^{\alpha}, \mathcal{H}_{\text {exch }}^{1 \mathrm{D}}\right]=0 \forall \alpha$. This implies the invariance of the generalized Heisenberg exchange Hamiltonian under the rotation operation in the spin space:

$$
\mathcal{H}^{\prime}{ }_{\text {exch }}^{1 \mathrm{D}}=\mathcal{H}_{\text {exch }}^{1 \mathrm{D}}
$$

being $\mathcal{H}^{\prime 1 \mathrm{D}}$ exch $=U_{R} \mathcal{H}_{\text {exch }}^{1 \mathrm{D}} U_{R}^{-1}$. Therefore, it has been rigorously proved that, in a quantum chain modeled by a generalized exchange Hamiltonian, the second-order biquadratic term does not break the rotational invariance of the exchange Hamiltonian. Straightforwardly, it can be shown that the inclusion of the energy term proportional to the external magnetic field or of an uniaxial anisotropy term breaks the rotational symmetry. This occurs because these terms fix a preferential spatial direction along which the spins of the $1 \mathrm{D}$ chain tend to align so that $\left[S^{\alpha}, \mathcal{H}^{1 \mathrm{D}}\right] \neq 0$. Therefore, the external field and the anisotropy fields are breaking symmetry terms of the generalized exchange Hamiltonian acting as ordering fields.

Analogously to the $1 \mathrm{D}$ case, it can be proved the invariance of the 2D generalized exchange Hamiltonian for a 2D quantum lattice of spins subjected to the periodic boundary conditions in the presence of the isotropic biquadratic exchange interaction. The 2D generalized exchange Hamiltonian for a quantum lattice can be written in the explicit form as:

$$
\mathcal{H}_{\mathrm{exch}}^{2 \mathrm{D}}=\sum_{i=0}^{N-1} \sum_{j=1}^{z / 2} J_{i j}^{(1)} \mathbf{S}_{i} \cdot \mathbf{S}_{j}+\sum_{i=0}^{N-1} \sum_{j=1}^{z / 2} J_{i j}^{(2)}\left(\mathbf{S}_{i} \cdot \mathbf{S}_{j}\right)^{2}
$$

where $z$ is the number of nearest neighbors with $z=1,2, \ldots$ depending on the lattice studied and the sum is over $z / 2$ to not count twice the interaction.

Starting from Equation (20) and following the same steps as for the 1D case it can be shown that:

$$
\left[S^{\alpha}, \mathcal{H}_{\text {exch }}^{2 \mathrm{D}}\right]=0
$$

that implies the invariance of the generalized exchange Hamiltonian for the 2D quantum lattice of $N$ spins under the rotation operation in the spin space:

$$
\mathcal{H}_{\text {exch }}^{2 \mathrm{D}}=\mathcal{H}_{\text {exch }}^{2 \mathrm{D}}
$$

\subsection{Absence of Long-Range Order for a 1D Chain of Spins Modeled via a Generalized Quantum Heisenberg Hamiltonian}

In this subsection, it is demonstrated the absence of long-range order for a 1D chain of spins described by the quantum Heisenberg Hamiltonian including both bilinear and biquadratic nearest neighbors exchange interactions extending the proof given by Mermin and Wagner for the bilinear [3]. The Bogoliubov inequality of Equation (6) takes the compact form:

$$
\left|m_{\boldsymbol{q}}\right|^{2} \leq \frac{1}{k_{B} T} S^{y y}(\boldsymbol{k}+\boldsymbol{q}) D_{1 \mathrm{D}}(\boldsymbol{k})
$$


with the double commutator $D_{1 \mathrm{D}}(k)$ given by:

$$
\begin{gathered}
D_{1 \mathrm{D}}(\boldsymbol{k})= \\
\frac{1}{N}\left(<\sum_{i=0}^{N-1} e^{-i \boldsymbol{k} \cdot \boldsymbol{R}_{i}} S_{i}^{x},\left[\left(\sum_{j=0}^{N-1} J_{j}^{(1)}{ }_{j+1} \mathbf{S}_{j} \cdot \mathbf{S}_{j+1}+\sum_{j=0}^{N-1} J_{j}^{(2)}\left(\mathbf{S}_{j+1} \cdot \mathbf{S}_{j+1}\right)^{2}\right), \sum_{l=0}^{N-1} e^{\left.\left.i \boldsymbol{k} \cdot \boldsymbol{R}_{l} S_{l}^{x} \delta_{l j+1}\right]\right]-}\right.\right. \\
B_{\mathrm{ext}}\left[\sum_{i=0}^{N-1} e^{-i} \boldsymbol{k} \cdot \boldsymbol{R}_{i} S_{i}^{x},\left[S_{\boldsymbol{q}}^{z}, \sum_{i=0}^{N-1} e^{i \boldsymbol{k} \cdot \boldsymbol{R}_{i}} S_{i}^{x}\right]\right]>
\end{gathered}
$$

with $\delta_{l j+1}=1$ for $l=j+1$ and $\delta_{l j+1}=0$ for $l \neq j+1$. The double commutator can be decomposed as $D_{1 \mathrm{D}}(\boldsymbol{k})=D_{1 \mathrm{D}}^{(1)}(\boldsymbol{k})+D_{1 \mathrm{D}}^{(2)}(\boldsymbol{k})+D_{1 \mathrm{D}}^{B_{\text {ext }}}(\boldsymbol{k})$ with $D_{1 \mathrm{D}}^{(1)}(\boldsymbol{k})\left(D_{1 \mathrm{D}}^{(2)}(\boldsymbol{k})\right)$ related to the bilinear (biquadratic) exchange contribution and $D_{1 \mathrm{D}}^{B_{\text {ext }}}(\boldsymbol{k})$ related to the term proportional to the external magnetic field contribution. In compact form:

$$
\begin{gathered}
D_{1 \mathrm{D}}^{(1)}(\boldsymbol{k})=\frac{1}{N} \sum_{i, j=0}^{N-1} e^{i \boldsymbol{k} \cdot\left(\boldsymbol{R}_{j+1}-\boldsymbol{R}_{i}\right)} J_{j j+1}^{(1)}\left\langle\left[S_{i}^{x},\left[\mathbf{S}_{j} \cdot \mathbf{S}_{j+1}, S_{j+1}^{x}\right]\right]\right\rangle \\
D_{1 \mathrm{D}}^{(2)}(\boldsymbol{k})=\frac{1}{N} \sum_{i, j=0}^{N-1} e^{i \boldsymbol{k} \cdot\left(\boldsymbol{R}_{j+1}-\boldsymbol{R}_{i}\right)} J_{j j+1}^{(2)}\left\langle\left[S_{i}^{x},\left[\left(\mathbf{S}_{j} \cdot \mathbf{S}_{j+1}\right)^{2}, S_{j+1}^{x}\right]\right]\right\rangle
\end{gathered}
$$

and:

$$
D_{1 \mathrm{D}}^{B_{\mathrm{ext}}}(\boldsymbol{k})=-\frac{1}{N} B_{\mathrm{ext}}<\left[\sum_{i=0}^{N-1} e^{-i \boldsymbol{k} \cdot \boldsymbol{R}_{i}} S_{i}^{x},\left[S_{\boldsymbol{q}}^{z} \sum_{i=0}^{N-1} e^{i k \cdot \boldsymbol{R}_{i}} S_{i}^{x}\right]\right]>
$$

where the rule $\langle A+B+\ldots\rangle=\langle A\rangle+\langle B\rangle+\ldots$ has been applied. After calculating the inner commutator on the second member of Equation (25) we get:

$$
D_{1 \mathrm{D}}^{(1)}(\boldsymbol{k})=i \frac{1}{N} \sum_{i, j=0}^{N-1} e^{i \boldsymbol{k} \cdot\left(\boldsymbol{R}_{j+1}-\boldsymbol{R}_{i}\right)}\left\langle\left[S_{i}^{x} \delta_{i(j, j+1)},\left(S_{j}^{z} S_{j+1}^{y}-S_{j}^{y} S_{j+1}^{z}\right)\right]\right\rangle
$$

Applying a commutator identity similar to Equation (18) to the inner commutator on the second member of Equation (26), after straightforward algebra the commutator takes the form:

$$
\begin{aligned}
{\left[\left(\mathbf{s}_{j} \cdot \mathbf{S}_{j+1}\right)^{2}, S_{j+1}^{x}\right]=} & \left(\left(S_{j+1}^{z}\right)^{2}-\left(S_{j+1}^{y}\right)^{2}\right)\left[S_{j}^{x},\left(S_{j}^{z}\right)^{2}\right]-\left(\left(S_{j}^{z}\right)^{2}-\left(S_{j}^{y}\right)^{2}\right)\left[S_{j+1}^{x},\left(S_{j+1}^{z}\right)^{2}\right]+ \\
& S_{j}^{x} S_{j+1}^{x}\left[\mathbf{S}_{j} \cdot \mathbf{S}_{j+1}, S_{j+1}^{x}\right]+\left[\mathbf{S}_{j} \cdot \mathbf{S}_{j+1}, S_{j+1}^{x}\right] S_{j}^{x} S_{j+1}^{x}
\end{aligned}
$$

with $\left[S_{l}^{x},\left(S_{l}^{z}\right)^{2}\right]=-i\left(S_{l}^{y} S_{l}^{z}+S_{l}^{z} S_{l}^{y}\right) l=j, j+1$. Hence:

$$
D_{1 \mathrm{D}}^{(2)}(\boldsymbol{k})=i \frac{1}{N} \sum_{i, j=0}^{N-1} e^{i \boldsymbol{k} \cdot\left(\boldsymbol{R}_{j+1}-\boldsymbol{R}_{i}\right)}\left\langle\left[S_{i}^{x} \delta_{i(j, j+1)}, f\left(S_{j}^{x}, S_{j+1}^{x}, S_{j}^{y}, S_{j+1}^{y}, S_{j}^{z}, S_{j+1}^{z}\right)\right]>\right.
$$

with:

$$
\begin{gathered}
f\left(S_{j}^{x}, S_{j+1}^{x}, S_{j}^{y}, S_{j+1}^{y}, S_{j}^{z}, S_{j+1}^{z}\right)=S_{j}^{x} S_{j+1}^{x}\left(S_{j}^{z} S_{j+1}^{y}-S_{j}^{y} S_{j+1}^{z}\right)+\left(S_{j}^{z} S_{j+1}^{y}-S_{j}^{y} S_{j+1}^{z}\right) S_{j}^{x} S_{j+1}^{x}- \\
\left(\left(S_{j+1}^{z}\right)^{2}-\left(S_{j+1}^{y}\right)^{2}\right)\left(S_{j}^{y} S_{j}^{z}+S_{j}^{z} S_{j}^{y}\right)+\left(\left(S_{j}^{z}\right)^{2}-\left(S_{j}^{y}\right)^{2}\right)\left(S_{j+1}^{y} S_{j+1}^{z}+S_{j+1}^{z} S_{j+1}^{y}\right)
\end{gathered}
$$


After calculating the inner commutator on the right side of Equation (27) yields:

$$
D_{1 \mathrm{D}}^{B_{\mathrm{ext}}}(\boldsymbol{k})=-\frac{i}{N} B_{\mathrm{ext}}<\sum_{i=0}^{N-1} e^{i \boldsymbol{q} \cdot \boldsymbol{R}_{i}}\left[S_{i}^{x}, S_{i}^{y}\right]>
$$

We get, via the calculation of the commutator, $D_{1 \mathrm{D}}^{B_{\mathrm{ext}}}(\boldsymbol{k})=\frac{1}{N} B_{\mathrm{ext}}<\sum_{i=0}^{N-1} e^{i} \boldsymbol{q} \cdot \boldsymbol{R}_{i} S_{i}^{z}>=\frac{1}{N} B_{\mathrm{ext}}<S_{\boldsymbol{q}}^{z}>=$ $B_{\text {ext }}\left|m_{q}\right|$ where the order parameter has been taken in modulus to represent a real quantity (see also the following arguments that justify this choice). As the commutator between two spin operators on different sites $(i \neq j, j+1)$ is zero, we get from Equation (28) for $i=j$ and $i=j+1$ :

$$
D_{1 \mathrm{D}}^{(1)}(\boldsymbol{k})=\frac{1}{N}\left\{\sum_{j=0}^{N-1} J_{j j+1}^{(1)}\left(\cos \left[\boldsymbol{k} \cdot\left(\boldsymbol{R}_{j}-\boldsymbol{R}_{j+1}\right)\right]-1\right)\left\langle S_{j}^{y} S_{j+1}^{y}+S_{j}^{z} S_{j+1}^{z}\right\rangle\right\}
$$

where the complex exponential of Equation (28) has been replaced by the cosine function due to $J_{i+1}$ ${ }^{(1)}=J_{i+1}{ }^{(1)}$. Analogously, from Equations (30) and (31) for $i=j$ and $i=j+1$, by applying some commutator identities, we obtain:

$$
\frac{1}{N}\left\{\sum_{j=0}^{N-1} J_{j j+1}^{(2)}\left(\cos \left[\boldsymbol{k} \cdot\left(\boldsymbol{R}_{j}-\boldsymbol{R}_{j+1}\right)\right]-1\right)\left(\begin{array}{c}
D_{1 \mathrm{D}}^{(2)}(\boldsymbol{k})= \\
\left.\left.\left\langle S_{j}^{x} S_{j+1}^{x}\left(S_{j}^{y} S_{j+1}^{y}+S_{j}^{z} S_{j+1}^{z}\right)+\left(S_{j}^{y} S_{j+1}^{y}+S_{j}^{z} S_{j+1}^{z}\right) S_{j}^{x} S_{j+1}^{x}\right\rangle\right)\right\rangle+ \\
\left.2\left(\begin{array}{c}
\left\langle\left(\left(S_{j}^{z}\right)^{2}-\left(S_{j}^{y}\right)^{2}\right)\left(\left(S_{j+1}^{z}\right)^{2}-\left(S_{j+1}^{y}\right)^{2}\right)\right\rangle+ \\
\left\langle\left(S_{j}^{y} S_{j}^{z}+S_{j}^{z} S_{j}^{y}\right)\left(S_{j+1}^{y} S_{j+1}^{z}+S_{j+1}^{z} S_{j+1}^{y}\right)\right\rangle
\end{array}\right)\right)
\end{array}\right)\right\}
$$

where the rule $\langle A \pm B+\ldots\rangle=\langle A\rangle \pm\langle B\rangle+\ldots$ has been applied.

Hence, the double commutator $D_{1 \mathrm{D}}(\boldsymbol{k})$ can be bounded by the following real and positive quantity:

$$
\begin{gathered}
D_{1 \mathrm{D}}(\boldsymbol{k})<B_{\mathrm{ext}}\left|m_{\boldsymbol{q}}\right|+\frac{|\boldsymbol{k}|^{2}}{2 N} \sum_{j=0}^{N-1}\left|J_{j j+1}^{(1)}\right|\left|\boldsymbol{R}_{j}-\boldsymbol{R}_{j+1}\right|^{2}\left|\left\langle\mathbf{S}_{j} \cdot \mathbf{S}_{j+1}\right\rangle\right|+ \\
\frac{|\boldsymbol{k}|^{2}}{2 N} \sum_{j=0}^{N-1}\left|J_{j j+1}^{(2)}\right|\left|\boldsymbol{R}_{j}-\boldsymbol{R}_{j+1}\right|^{2}\left\langle\left(\mathbf{S}_{j} \cdot \mathbf{S}_{j+1}\right)^{2}\right\rangle \leq B_{\mathrm{ext}}\left|m_{\boldsymbol{q}}\right|+S(S+1) \bar{J}^{(1)}|\boldsymbol{k}|^{2}+S^{2}(S+1)^{2} \bar{J}^{(2)}|\boldsymbol{k}|^{2}
\end{gathered}
$$

where $|\boldsymbol{k}|^{2}=k^{2},\left|\boldsymbol{R}_{j}-\boldsymbol{R}_{j+1}\right|^{2}=\left(R_{j}-R_{j+1}\right)^{2},\left|\boldsymbol{R}_{j}-\boldsymbol{R}_{j+1}\right|$ is the spin nearest-neighbors distance, $\bar{J}^{(1)}=\frac{1}{N} \sum_{j=0}^{N-1}\left|J_{j j+1}^{(1)}\right|\left|\boldsymbol{R}_{j}-\boldsymbol{R}_{j+1}\right|^{2}<\infty$ and $\bar{J}^{(2)}=\frac{1}{N} \sum_{j=0}^{N-1}\left|J_{j j+1}^{(2)}\right|\left|\boldsymbol{R}_{j}-\boldsymbol{R}_{j+1}\right|^{2}<\infty$, two conditions for short-range exchange interactions.

In the first inequality (35) a small and positive upper bound to the difference $\left(\cos \left[k \cdot\left(\boldsymbol{R}_{j}-\boldsymbol{R}_{j+1}\right)\right]\right.$ - 1) (Equations (33) and (34)), that is at most equal to zero, proportional to $|\boldsymbol{k}|^{2} / 2\left|\boldsymbol{R}_{j}-\boldsymbol{R}_{j+1}\right|^{2}$ has been chosen within the framework of short-range exchange interactions. Hence, the upper bound of the first inequality can be understood taking into account that, for the bilinear term, it is $D_{1 \mathrm{D}}^{(1)}(\boldsymbol{k})<\frac{|\boldsymbol{k}|^{2}}{2 N} \sum_{j=0}^{N-1}\left|J_{j}^{(1)}\right|\left|\boldsymbol{R}_{j}-\boldsymbol{R}_{j+1}\right|^{2}\left|\left\langle\mathbf{S}_{j} \cdot \mathbf{S}_{j+1}\right\rangle\right|$, where the last factor comes from $\left|\left\langle S_{j}^{y} S_{j+1}^{y}+S_{j}^{z} S_{j+1}^{z}\right\rangle\right| \leq\left|\left\langle\mathbf{S}_{j} \cdot \mathbf{S}_{j+1}\right\rangle\right|$, while, following a similar argument, for the biquadratic term, it is $D_{1 \mathrm{D}}^{(2)}(\boldsymbol{k})<\frac{|\boldsymbol{k}|^{2}}{2 N} \sum_{j=0}^{N-1}\left|J_{j j+1}^{(2)}\right|\left|\boldsymbol{R}_{j}-\boldsymbol{R}_{j+1}\right|^{2}\left\langle\left(\mathbf{S}_{j} \cdot \mathbf{S}_{j+1}\right)^{2}\right\rangle$.

The upper bounds of the second inequality of Equation (35) are, for $D_{1 \mathrm{D}}^{(1)}(\boldsymbol{k})$, the maximum eigenvalues of the operators $\left(\mathbf{S}_{j}+\mathbf{S}_{j+1}\right)^{2}$ and $\mathbf{S}_{i}{ }^{2}$ related to the bilinear interaction so that $\left|\left\langle\mathbf{S}_{j} \cdot \mathbf{S}_{j+1}\right\rangle\right| \leq S(S+1)$, while, for $D_{1 \mathrm{D}}^{(2)}(\boldsymbol{k})$, the maximum eigenvalues of the operators $\left(\mathbf{S}_{j}+\mathbf{S}_{j+1}\right)^{4}$ 
and $\mathbf{S}_{i}{ }^{4}$ related to the biquadratic contribution so that $\left\langle\left(\mathbf{S}_{j} \cdot \mathbf{S}_{j+1}\right)^{2}\right\rangle \leq S^{2}(S+1)^{2}$. Taking into account Equations (23) and (35), the following strict inequality in terms of the order parameter holds:

$$
\left|m_{\boldsymbol{q}}\right|^{2}<\frac{1}{k_{B} T} S^{y y}(\boldsymbol{k}+\boldsymbol{q})\left\{B_{\text {ext }}\left|m_{\boldsymbol{q}}\right|+S(S+1) \bar{J}^{(1)}|\boldsymbol{k}|^{2}+S^{2}(S+1)^{2} \bar{J}^{(2)}|\boldsymbol{k}|^{2}\right\}
$$

By summing over the wave vectors of the 1D Brillouin zone (sum of momenta) and by taking into account that $S(S+1) \geq \frac{1}{N} \sum_{i=0}^{N-1}\left\langle\left(S_{i}^{y}\right)^{2}\right\rangle=\frac{1}{N} \sum_{\boldsymbol{k}} S^{y y}(\boldsymbol{k}+\boldsymbol{q})$, the inversion of the inequality yields:

$$
S(S+1)>\frac{k_{B} T\left|m_{\boldsymbol{q}}\right|^{2}}{N} \sum_{k}\left[\frac{1}{B_{\mathrm{ext}}\left|m_{\boldsymbol{q}}\right|^{2}+S(S+1) \bar{J}^{(1)}|\boldsymbol{k}|^{2}+S^{2}(S+1)^{2} \bar{J}^{(2)}|\boldsymbol{k}|^{2}}\right]
$$

Converting, in the thermodynamic limit $(N \rightarrow \infty)$, the sum of momenta into a 1D integral yields:

$$
S(S+1)>\frac{k_{B} T L}{2 \pi}\left|m_{\boldsymbol{q}}\right|^{2} \int_{0}^{\bar{k}} \frac{d k}{B_{\text {ext }}\left|m_{\boldsymbol{q}}\right|+S(S+1) \bar{J}^{(1)}|\boldsymbol{k}|^{2}+S^{2}(S+1)^{2} \bar{J}^{(2)}|\boldsymbol{k}|^{2}}
$$

where $L$ is the length of the 1D unit cell and $\bar{k}<k_{1 \mathrm{BZ}}$ with $k_{1 \mathrm{BZ}}$ the edge wave vector of the first Brillouin zone (1BZ). The Mermin and Wagner result [3,28] is easily obtained by setting $J_{j j+1}^{(2)}=0 \forall j$ implying $\bar{J}^{(2)}=0$.

We now consider the general case $J_{j j+1}^{(1)} \neq 0$ and $J_{j j+1}^{(2)} \neq 0$ with $2 J_{i i+1}^{(2)}>\left|J_{i i+1}^{(1)}\right|$ that can occur for quantum chains characterized by a generalized exchange Hamiltonian. This case corresponds to a $C$ ground state where adjacent spins form any angles $\varphi_{i i+1}$ one over the other. By solving the integral of Equation (38) and by inverting the inequality leads to:

$$
\left|m_{q}\right|^{\frac{3}{2}}<\frac{2 \pi S(S+1)^{\frac{3}{2}}}{k_{B} T L} \frac{\left(\left(\bar{J}^{(1)}+S(S+1) \bar{J}^{(2)}\right) B_{\text {ext }}\right)^{\frac{1}{2}}}{\arctan \left(\bar{k}\left(S(S+1)\left(\bar{J}^{(1)}+S(S+1) \bar{J}^{(2)}\right) /\left(B_{\text {ext }}\left|m_{q}\right|\right)\right)^{\frac{1}{2}}\right)}
$$

In the limit of small $B_{\text {ext }} \arctan (x)$ with $x=\bar{k}\left(S(S+1)\left(\bar{J}^{(1)}+S(S+1) \bar{J}^{(2)}\right) /\left(B_{\text {ext }}\left|m_{q}\right|\right)\right)^{\frac{1}{2}}$ is replaced by $\pi / 2$ yielding:

$$
\left|m_{q}\right|<f_{1 \mathrm{D}}\left(\bar{J}^{(1)}, \bar{J}^{(2)}, T, S\right)\left(B_{\mathrm{ext}}\right)^{\frac{1}{3}}
$$

where:

$$
f_{1 \mathrm{D}}\left(\bar{J}^{(1)}, \bar{J}^{(2)}, T, S\right)=\frac{2^{\frac{4}{3}}(S(S+1))\left(\bar{J}^{(1)}+S(S+1) \bar{J}^{(2)}\right)^{\frac{1}{3}}}{\left(k_{\mathrm{B}} T\right)^{\frac{2}{3}} L^{\frac{2}{3}}}
$$

with $\bar{J}^{(1)}=\bar{J}^{(1)}(N), \bar{J}^{(2)}=\bar{J}^{(2)}(N)$.

The power law for the order parameter of Equation (40) referred to the generalized quantum Heisenberg Hamiltonian is the same as for the 1D Heisenberg Hamiltonian characterized solely by the bilinear term and the coefficient $f_{1 \mathrm{D}}$ includes in addition the dependence on the biquadratic term.

Starting from the double commutator of the 2D ferromagnetic lattice system:

$$
D_{2 \mathrm{D}}(\boldsymbol{k})=\frac{1}{N}<\left[\sum_{i=0}^{N-1} e^{-i} \boldsymbol{k} \cdot \boldsymbol{R}_{i} S_{i}^{x},\left[\left(\sum_{j=0}^{N-1} \sum_{l=1}^{z / 2} J_{j l}^{(1)} \mathbf{S}_{j} \cdot \mathbf{S}_{l}+\sum_{j=0}^{N-1} \sum_{l=1}^{z / 2} J_{j l}^{(2)}\left(\mathbf{S}_{j} \cdot \mathbf{S}_{l}\right)^{2}-B_{\mathrm{ext}} S_{q}^{z}\right), \sum_{l=0}^{N-1} e^{i \boldsymbol{k} \cdot \boldsymbol{R}_{l}} S_{l}^{x}\right]\right]>
$$


and following, for the 2D quantum ferromagnetic lattice, the same steps done for the 1D chain of quantum spins, the inequality of Equation (38) is replaced by:

$$
S(S+1)>\frac{k_{B} T A}{(2 \pi)^{2}}\left|m_{\boldsymbol{q}}\right|^{2} \int_{0}^{\bar{k}} \frac{k d k}{B_{\mathrm{ext}}\left|m_{\boldsymbol{q}}\right|+S(S+1) \bar{J}^{(1)}|\boldsymbol{k}|^{2}+S^{2}(S+1)^{2} \bar{J}^{(2)}|\boldsymbol{k}|^{2}} \int_{0}^{2 \pi} d \varphi
$$

where $A$ is the area of the surface of the generic $2 \mathrm{D}$ spin lattice and the integration is over a circular region ( $\varphi$ is the azimuthal angle) of the $2 \mathrm{D}$ reciprocal lattice of radius $\bar{k}$ with $\bar{k}<k_{1 \mathrm{BZ}}$. Here, $k_{1 \mathrm{BZ}}$ is the edge wave vector of the $1 \mathrm{BZ}$ zone. The integration on the second member and the inversion of the inequality yields:

$$
\left|m_{q}\right|^{2}<4 \pi \frac{S^{2}(S+1)^{2}\left(\bar{J}^{(1)}+S(S+1) \bar{J}^{(2)}\right)}{k_{B} T A \ln \left(1+\bar{k}^{2} \frac{S(S+1)\left(\bar{J}^{(1)}+S(S+1) \bar{J}^{(2)}\right)}{B_{\text {ext }}\left|m_{q}\right|}\right)}
$$

In the limit of small $B_{\mathrm{ext}}, \ln (1+\mathrm{x})$, with $x=C / B_{\mathrm{ext}}$ and $C=\bar{k}^{2} \frac{S(S+1)\left(\bar{J}^{(1)}+S(S+1) \bar{J}^{(2)}\right)}{\left|m_{q}\right|}$, is approximated by $\ln x$ being $x>>1$. For $x \rightarrow \infty, \ln \left(C / B_{\text {ext }}\right) \rightarrow \infty$ and can be replaced by $\left|\ln \left(C B_{\text {ext }}\right)\right|$ having the same behavior. By virtue of these approximations, relation of Equation (44) can be rewritten as:

$$
\left|m_{q}\right|<f_{2 \mathrm{D}}\left(\bar{J}^{(1)}, \bar{J}^{(2)}, T, S\right) \frac{1}{\left(\left|\ln \left(C B_{\text {ext }}\right)\right|\right)^{\frac{1}{2}}}
$$

where:

$$
f_{2 \mathrm{D}}\left(\bar{J}^{(1)}, \bar{J}^{(2)}, T, S\right)=\frac{2(\pi)^{\frac{1}{2}} S(S+1)\left(\bar{J}^{(1)}+S(S+1) \bar{J}^{(2)}\right)^{\frac{1}{2}}}{\left(k_{\mathrm{B}} T\right)^{\frac{1}{2}} A^{\frac{1}{2}}}
$$

The law expressing the dependence on $B_{\text {ext }}$ given by Equation (45) is the same as for the $2 \mathrm{D}$ quantum Heisenberg Hamiltonian characterized solely by the bilinear term and the only effect of the biquadratic term is to change the coefficient $C$ in front of $B_{\text {ext }}$ and the coefficient $f_{2 \mathrm{D}}$.

According to the inequalities of Equations (40) and (45) valid for 1D and 2D quantum ferromagnetic systems, respectively, it is always, both for the 1D quantum chain and 2D spin lattice with $N$ spins, $\forall T \neq 0$ :

$$
\lim _{N \rightarrow \infty}\left[\lim _{B_{\text {ext }} \rightarrow 0^{+}}\left[\left|m_{\boldsymbol{q}}\left(B_{\text {ext }}, N\right)\right|\right]\right]=0
$$

where the outer limit refers to the thermodynamic limit. The result of Equation (47) means that there is no long-range order at any $T \neq 0$ for dimensionality $d=1,2$ in quantum spin ferromagnetic systems whose exchange Hamiltonian includes both the isotropic bilinear and biquadratic exchange terms. According to the result of Equation (47), F ground-state, AF ground-state, and C ground-state arising either from $\mathrm{F}$ or $\mathrm{AF}$ ground-state, appearing in quantum spin ferromagnetic systems modeled by a generalized quantum Heisenberg Hamiltonian are ruled out at any finite $T$ for $d \leq 2$.

In this way, a generalization of the Hohenberg-Mermin-Wagner theorem has been obtained showing that, for $1 \mathrm{D}$ and $2 \mathrm{D}$ ferromagnetic quantum spin systems modeled via a generalized quantum Heisenberg Hamiltonian, the order parameter: (1) vanishes as the external magnetic field tends to zero and (2) fulfils the same laws as for the quantum Heisenberg Hamiltonian characterized by only the bilinear exchange term. Taking into account the underlying physics, it is demonstrated that there is absence of long-range order at any $T \neq 0$ when the quantum spins are also in a $C$ ground-state and, also in this case, there is no spontaneous spin symmetry breaking. The absence of long-range order in these systems does not exclude the occurrence of any kinds of phase transitions as found in [20] at finite $T$ characterized by the divergence of the susceptibility for vanishing external magnetic field 
analogously to what occurred for the Mermin and Wagner case. Finally, note that the continuous rotational symmetry of the generalized quantum exchange Hamiltonian not only implies its spatial invariance under the spin rotation operation for the 1D and 2D systems, respectively (Equations (19) and (22)), but also the absence of spontaneous spin symmetry breaking.

\section{Results and Discussion}

In this Section, the main results obtained for the 1D and 2D systems are discussed in more detail. These results are related to the biquadratic interaction that has been incorporated into the exchange Hamiltonian leading to a generalized second-order exchange Hamiltonian. The necessity of invoking a biquadratic exchange interaction, in addition to a bilinear exchange interaction, to explain the energy level spectrum of Mn pairs in $\mathrm{MgO}$ [13] led to the investigation of the origin of this novel interaction in terms of superexchange interaction $[15,29,30]$. From the exchange energy minimization, this would ideally correspond to a canted state between the electron spins of Mn pairs for comparable magnitudes of the bilinear and biquadratic interactions.

Later, the existence of this type of interaction was confirmed to interpret the experimental data based on magneto optic Kerr effect microscopy, magnetization curves and domain observations in $\mathrm{Fe} / \mathrm{Cr} / \mathrm{Fe}$ ferromagnetic bilayers $[18,31]$ and several other ferromagnetic bilayer systems with different paramagnetic spacers (e.g., $\mathrm{Fe} / \mathrm{Au} / \mathrm{Fe}, \mathrm{Fe} / \mathrm{Al} / \mathrm{Fe}, \mathrm{Co} / \mathrm{Cu} / \mathrm{Co}$, ect.) [18]. In these systems characterized also by a non-negligible crystallographic anisotropy the biquadratic exchange interaction favors the $90^{\circ}$ ground-state between two adjacent ferromagnetic layers. On the other hand, there are also ferromagnetic bilayers such as $\mathrm{FeNi} / \mathrm{Ag} / \mathrm{FeNi}$ where, due to negligible crystallographic anisotropy, the biquadratic interaction favors a canted phase between the two magnetization of coupled ferromagnetic layers when $2\left|J^{(2)}\right|>\left|J^{(1)}\right|$ with $J^{(1)}$ and $J^{(2)}$ the bilinear and biquadratic exchange constants, respectively. The biquadratic exchange interaction together with the higher-order exchange interactions were theoretically interpreted as arising from the spin-asymmetry of the electron reflection amplitudes at the ferromagnetic-paramagnetic interfaces. This modelization was used to describe phenomenologically the interlayer exchange coupling between ferromagnetic layers separated by a paramagnetic spacer $[17,18]$.

The presence of the biquadratic interaction enriches the phase diagram of these quantum systems adding new ground states besides the ferro-and anti-ferromagnetic ground states. This depends on the relative sign and magnitude of the bilinear and biquadratic nearest-neighbors interactions. According to the Hamiltonian expressed in Equation (2) in zero external magnetic field the case $2 J_{i j}^{(2)}>\left|J_{i j}^{(1)}\right|$ is analyzed. This is the most interesting case because this state corresponds to the so-called $C$ ground-state where adjacent spins form equilibrium angles $0<\varphi_{i j}<\pi$. Minimizing the generalized quantum spin exchange Hamiltonian for $1 \mathrm{D}$ and $2 \mathrm{D}$ systems yields $\forall i, j$ :

$$
\varphi_{i j}=\arccos \left[-\frac{J_{i j}^{(1)}}{2 J_{i j}^{(2)}}\right]
$$

Figure 1 summarizes the ground states of a 1D chain of quantum fractional integer spins modeled by means of a generalized quantum Heisenberg Hamiltonian that are attached to the ferromagnetic atoms of the chain for $B_{\mathrm{ext}}=0$. Figure $1 \mathrm{a}, \mathrm{b}$ display the $\mathrm{F}$ and AF ground-state, obtained for $J_{i+1}^{(1)}<0$ and $J_{i+1}^{(2)}=0$ and $J_{i+1}^{(1)}>0$ and $J_{i+1}^{(2)}=0$, respectively. The AF ground-state forms considering two spin sublattices. Figure $1 \mathrm{c}, \mathrm{d}$ show the $\mathrm{C}$ ground-state forming from a $\mathrm{F}$ ground-state for $J_{i+1}^{(1)}<0$ and $J_{i i+1}^{(2)}>0\left(0<\varphi_{i i+1}<\pi / 2\right)$ and from an AF ground-state for $J_{i i+1}^{(1)}>0$ and $J_{i+1}^{(2)}>0$ $\left(\pi / 2<\varphi_{i i+1}<\pi\right)$, respectively. We call the former the C-F ground-state and the latter the C-AF ground state. In each state the amplitudes of equilibrium angles $\varphi_{i i+1}$ are different depending on the couple of spin operators considered. 

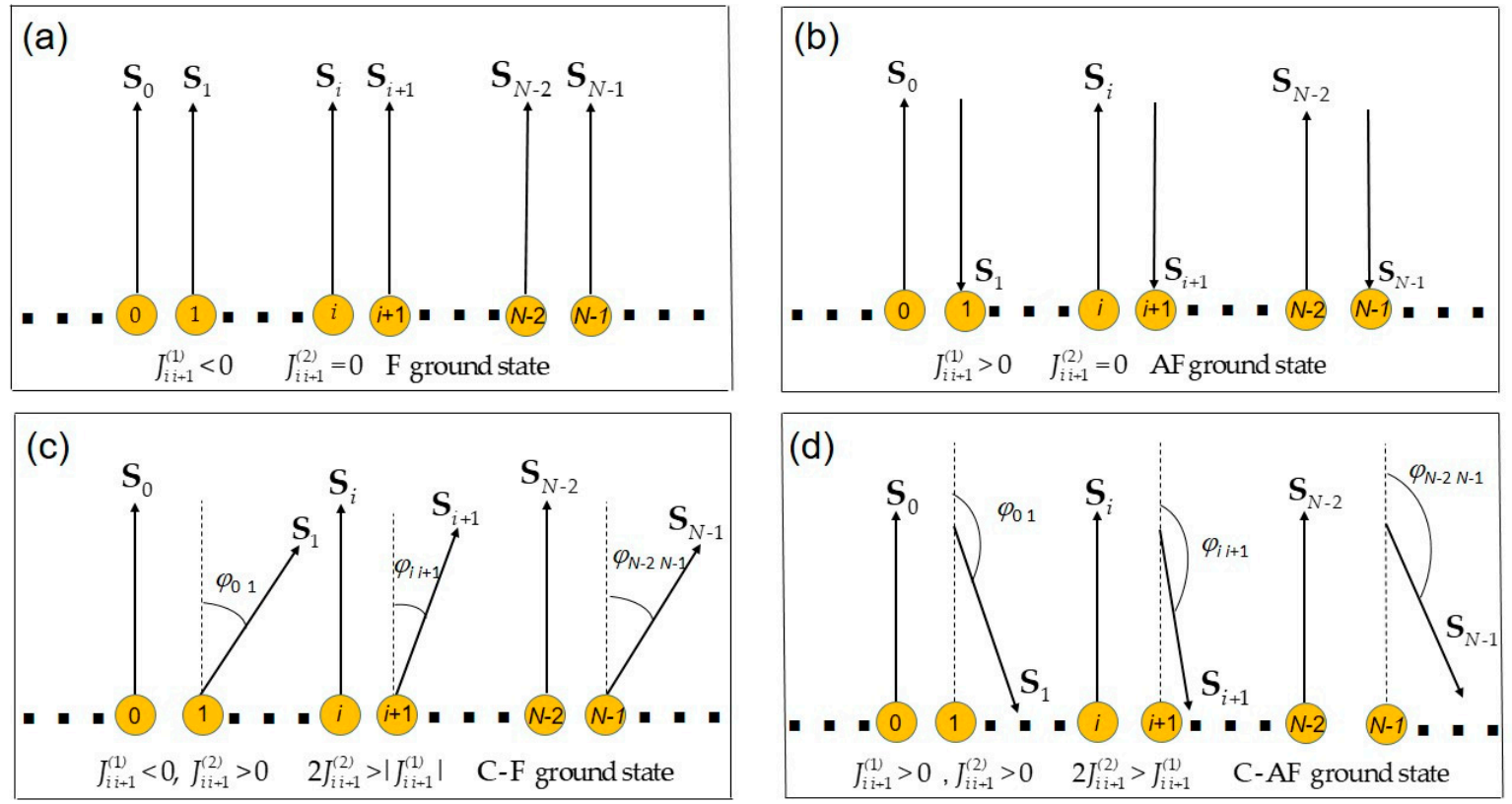

Figure 1. Quantum chain ground states with the spin vector operators $\mathbf{S}_{i}(i=0,2, \ldots N-1)$ ideally attached to the ferromagnetic atoms represented with brown circles subjected to periodic boundary conditions. The lattice parameter of the chain is $L$ and corresponds to the spin nearest-neighbors distance. (a) F ground state $\left(\varphi_{i i+1}=0 \forall i\right)$. (b) AF ground-state $\left(\varphi_{i+1}=\pi \forall i\right)$. (c) C-F ground-state for $J_{i+1}^{(2)}>0$. (d) C-AF ground-state for $J_{i+1}^{(2)}>0$.

For $J_{i+1}^{(2)}>>\left|J_{i+1}^{(1)}\right| \varphi_{i+1} \rightarrow \pi / 2$ so that the adjacent spins would ideally form a right angle one with respect to the other independently of the relative sign of $J_{i i+1}^{(1)}$ and $J_{i+1}^{(2)}$. With increasing the external magnetic field amplitude with the magnetic field along $+z$, the equilibrium angles $\varphi_{i i+1}$ reduce their amplitudes $\forall i$ both for C-F and C-AF states.

With increasing $B_{\text {ext }}$ with the external field along the $z$-axis, the angles $\varphi_{i+1}$ progressively reduce their amplitude and, for high $B_{\text {ext, }}$ the $\boldsymbol{S}_{i}$ spins become parallel one to each other aligning along the $z$-direction $\left(\varphi_{i+1} \rightarrow 0 \forall i\right)$. The same conclusions can be drawn to the $2 \mathrm{D}$ quantum spin ferromagnetic lattice of any type (square, rectangular, rhombic, hexagonal, ... ) provided that $i+1$ is replaced by the number $z$ of first neighbors depending on the lattice considered.

Finally, it is interesting to discuss practically the approximation of the spin rotation operator (Equation (15)) used to prove the invariance of the 1D generalized exchange Hamiltonian $\mathcal{H}_{\text {exch }}^{1 \mathrm{D}}$ under a continuous spatial rotation in the spin space. Indeed, $\mathcal{H}_{\text {exch }}^{1 \mathrm{D}}$ Hamiltonian models the chain spin operators displayed in Figure 1 and their relative equilibrium configurations.

For spin systems, it can be defined, as is usual for quantum mechanical rotation operators [32], a spin rotation operator $U_{R}(\hat{\boldsymbol{n}}, \theta)=e^{-i \theta \hat{\boldsymbol{n}} \cdot \mathbf{S}}$ (the spin rotation generator $\mathbf{S}$ is dimensionless in our representation) associated to a continuous spatial rotation $R$ in the spin space. To prove that the generalized exchange Hamiltonian of the 1D chain of spins, as the one shown in Figure 1, is rotationally invariant, one should, in principle, demonstrate that $U_{R}(\hat{\boldsymbol{n}}, \theta)$ commutes with $\mathcal{H}_{\text {exch. }}^{1 \mathrm{D}}$. However, to prove this invariance, it is enough to take into account only the first-order series expansion, $U_{R}(\hat{\boldsymbol{n}}, \theta) \approx 1-i \hat{\boldsymbol{n}} \cdot \mathbf{S}$ (Equation (15)). This would practically correspond to consider infinitesimal angles $\delta \theta$ in the rotation operation. Indeed, if one includes the higher-order terms of the expansion of the spin rotation generator, some commutator identities involving the product of operators can be applied. In this way, all terms can be rewritten in terms of commutators between first-order spin generators that cancel out two by two leading to $\left[U_{R}(\hat{\boldsymbol{n}}, \theta), \mathcal{H}_{\text {exch }}^{1 \mathrm{D}}\right]=0$. The same arguments can be applied to the $2 \mathrm{D}$ ferromagnetic lattices of quantum fractional integer spins modeled by the Hamiltonian $\mathcal{H}_{\text {exch }}^{2 D}$ of Equation (20) leading to $\left[U_{R}(\hat{\boldsymbol{n}}, \theta), \mathcal{H}_{\text {exch }}^{2 \mathrm{D}}\right]=0$. 


\section{Conclusions}

In summary, in this paper the Hohenberg-Mermin-Wagner theorem was generalized for 1D and 2D quantum ferromagnetic systems modeled through a generalized quantum Heisenberg Hamiltonian including isotropic bilinear and biquadratic interactions. This generalization was accomplished by applying statistical arguments based on the use of the Bogoliubov inequality and of the double commutator function. It has been shown that the generalized quantum Heisenberg Hamiltonian of a 1D ferromagnetic quantum chain and of a 2D quantum ferromagnetic lattice of fractional integer spins with nearest-neighbors interaction does not allow long-range order at any finite temperature $T$ and for any type of bilinear and biquadratic exchange coupling. The on-site magnetization vanishes with the vanishing of the ordering field according to the same law as for the $1 \mathrm{D}$ and 2D quantum Heisenberg Hamiltonian where only the bilinear interaction was taken into account. Hence, not only ferromagnetism and antiferromagnetism are ruled out as occurs in the Mermin and Wagner case but also a canting ground-state resulting from the balancing between the isotropic bilinear and biquadratic interactions is ruled out. The canting ground-state can result either from the ferromagnetic or from the antiferromagnetic ground-state depending on the sign of the bilinear exchange term. It has also been shown that the rotational symmetry under the spin rotation generator proved for the generalized exchange Hamiltonian in both 1D and 2D systems of quantum spins implies the absence of spontaneous symmetry breaking at finite temperature. On the other hand, the 1D and 2D Hamiltonians are not anymore rotationally invariant in the presence of an anisotropy contribution leading to a spontaneous symmetry breaking for $T \neq 0$. These results could advance the understanding of continuous symmetries in quantum magnetic systems.

Funding: This research received no external funding.

Acknowledgments: The support from the group of Mathematical Physics (INdAM) is gratefully acknowledged.

Conflicts of Interest: The author declares no conflict of interest.

\section{References}

1. Chaikin, P.M.; Lubenski, T.C. Principles of Condensed Matter Physics, 1st ed.; Cambridge University Press: Cambridge, UK, 2012.

2. Hohenberg, P.C. Existence of long-range order in one- and two-dimensions. Phys. Rev. 1967, 158, 383-386. [CrossRef]

3. Mermin, N.D.; Wagner, H. Absence of ferromagnetism or anti-ferromagnetism in one- or two-dimensional Heisenberg models. Phys. Rev. Lett. 1966, 17, 1133-1136. [CrossRef]

4. Halperin, B.E. On the Hohenberg-Mermin-Wagner Theorem and Its Limitations. J. Stat. Phys. 2018, 175, 521-529. [CrossRef]

5. Coleman, S. There are no Goldstone Bosons in Two Dimensions. Commun. Math. Phys. 1973, 31, 259-264. [CrossRef]

6. Bogoliubov, N.N. Quasimittelwerte in Problemen der statistischen Mechanik (1. Fortsetzung). Phys. Abhandl. Sowjetunion 1962, 6, 1 .

7. Mermin, N.D. Absence of Ordering in Certain Classical Systems. J. Math. Phys. 1967, 8, 1061-1064. [CrossRef]

8. Gelfert, A.; Nolting, W. Absence of a Magnetic Phase Transition in Heisenberg, Hubbard, and Kondo-Lattice (s-f) Films. Phys. Status Solidi 2000, 217, 805-818. [CrossRef]

9. Gelfert, A.; Nolting, W. The absence of finite-temperature phase transitions in low-dimensional many-body models: A survey and new results. J. Phys. Cond. Matter 2001, 13, R505. [CrossRef]

10. Walker, M.B. Nonexistence of excitonic insulators in one and two dimensions. Can. J. Phys. 1968, 46, 817. [CrossRef]

11. Bruno, P. Absence of Spontaneous Magnetic Order at Nonzero Temperature in One- and Two-Dimensional Heisenberg and $X Y$ Systems with Long-Range Interactions. Phys. Rev. Lett. 2001, 87, 187203. [CrossRef] [PubMed] 
12. Loss, D.; Pedrocchi, F.L.; Leggett, A.J. Absence of Spontaneous Magnetic Order of Lattice Spins Coupled to Itinerant Interacting Electrons in One and Two Dimensions. Phys. Rev. Lett. 2011, 107, 107201. [CrossRef] [PubMed]

13. Harris, E.A.; Owen, J. Biquadratic Exchange Between $\mathrm{Mn}^{2+}$ Ions in MgO. Phys. Rev. Lett. 1963, 11, 9. [CrossRef]

14. Rodbell, D.S.; Jacobs, I.S.; Owen, J.; Harris, E.A. Biquadratic Exchange and the Behavior of Some Antiferromagnetic Substances. Phys. Rev. Lett. 1963, 11, 10. [CrossRef]

15. Huang, N.L.; Orbach, R. Biquadratic Superexchange. Phys. Rev. Lett. 1964, 12, 275. [CrossRef]

16. Chen, H.H.; Levy, P. High-Temperature Series Expansion for a spin-1 Model of Ferromagnetism. Phys. Rev. B 1973, 7, 4284. [CrossRef]

17. Bruno, P. Interlayer exchange coupling: A unified physical picture. J. Magn. Magn. Mater. 1993, 121, $248-252$. [CrossRef]

18. Demokritov, J. Biquadratic interlayer coupling in layered magnetic systems. J. Phys. D Appl. Phys. 1998, 31, 925-941. [CrossRef]

19. Strässle, T.; Juranyi, F.; Schneider, M.; Janssen, S.; Furrer, A.; Krämer, K.W.; Güdel, H.U. Origin of Higher Order Magnetic Exchange: Evidence for Local Dimer Exchange Striction in $\mathrm{CsMn}_{0.28} \mathrm{Mg}_{0.72} \mathrm{Br}_{3}$ Probed by Inelastic Neutron Scattering. Phys. Rev. Lett. 2004, 92, 257252. [CrossRef]

20. Stanley, H.E.; Kaplan, T.A. Possibility of a Phase Transition for the Two-Dimensional Heisenberg Model. Phys. Rev. Lett. 1966, 17, 913. [CrossRef]

21. Mattis Daniel, C. The Theory of Magnetism II-Thermodynamics and Statistical Mechanics; Springer: Berlin/Heidelberg, Germany, 1985.

22. Frölich, J.; Spencer, T. Kosterlitz-Thouless Transition in the Two-Dimensional Plane Rotator and Coulomb Gas. Phys. Rev. Lett. 1966, 46, 1006. [CrossRef]

23. Frölich, J.; Lieb, E.H. Phase transitions in anisotropic lattice spin systems. Commun. Math. Phys. 1978, 60, $233-267$. Available online: https://projecteuclid.org:443/euclid.cmp/1103904129 (accessed on 18 November 2020). [CrossRef]

24. Thorpe, M.F. Absence of Ordering in Certain Isotropic Systems. J. Appl. Phys 1971, 42, 1410-1411. [CrossRef]

25. Krzemiński, S. Note on the Mermin-Wagner Theorem. Phys. Status Solidi 1976, 14, K119-K123. [CrossRef]

26. Suzuki, M. Long-Range Order in Ideal Ferromagnets. Prog. Theor. Phys. 1969, 42, 1086-1907. [CrossRef]

27. Frölich, J.; Pfister, C. On the Absence of Spontaneous Symmetry Breaking and of Crystalline Ordering in Two-Dimensional Systems. Commun. Math. Phys. 1981, 81, 277-298. Available online: https://projecteuclid. org/euclid.cmp/1103920246 (accessed on 18 November 2020). [CrossRef]

28. Auerbach, A. Interacting Electrons and Quantum Magnetism; Springer: New York, NY, USA, 1994.

29. Anderson, P.H. New Approach to the Theory of Superexchange Interactions. Phys. Rev. 1959, 115, 2. [CrossRef]

30. Anderson, P.H. Theory of Magnetic Exchange Interactions: Exchange in Insulators and Semiconductors. In Solid State Physics; Seitz, F., Turnbull, D., Eds.; Academic Press, Inc.: New York, NY, USA, 1963; Volume 14, pp. 99-214. [CrossRef]

31. Rührig, M.; Schäfer, R.; Hubert, A.; Mosler, R.; Wolf, J.A.; Demokritov, S.O.; Grünberg, P. Domain Observations on Fe-Cr-Fe Layered Structures. Evidence for a Biquadratic Coupling Effect. Phys. Status Solidi 1991, 125, 635. [CrossRef]

32. Landau, L.D.; Lifshitz, E.M. Quantum Mechanics: Non-Relativistic Theory, 3rd ed.; Elsevier: Amsterdam, The Netherlands, 1981; Volume 3.

Publisher's Note: MDPI stays neutral with regard to jurisdictional claims in published maps and institutional affiliations.

(C) 2020 by the author. Licensee MDPI, Basel, Switzerland. This article is an open access article distributed under the terms and conditions of the Creative Commons Attribution (CC BY) license (http://creativecommons.org/licenses/by/4.0/). 